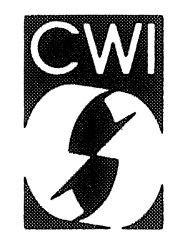

Centrum voor Wiskunde en Informatica Centre for Mathematics and Computer Science

O.J. Boxma, J.W. Cohen

The M/G/1 queue with permanent customers 
The Centre for Mathematics and Computer Science is a research institute of the Stichting Mathematisch Centrum, which was founded on February 11, 1946 , as a nonprofit institution aiming at the promotion of mathematics, computer science, and their applications. It is sponsored by the Dutch Government through the Netherlands Organization for the Advancement of Research (N.W.O.).

Copyright $(\mathcal{C}$ Stichting Mathematisch Centrum, Amsterdam 


\title{
The M/G/1 Queue with Permanent Customers
}

\author{
O.J. Boxma, J.W. Cohen \\ Centre for Mathematics and Computer Science \\ P.O. Box 4079, 1009 AB Amsterdam, The Netherlands
}

\begin{abstract}
This paper is concerned with an M/G/1 FCFS queue with two types of customers, viz. (i) ordinary customers who arrive according to a Poisson process, and (ii) permanent customers, who immediately return to the end of the queue after having received a service. The influence of the permanent customers on queue length and sojourn times of the Poisson customers is studied, using results from queueing theory and from the theory of branching processes. In particular it is shown, for the case that the service time distributions of the Poisson customers and all $K$ permanent customers are negative exponential with identical means, that the queue length and sojourn time distributions of the Poisson customers are the $(K+1)$-fold convolution of those for the case without permanent customers.
\end{abstract}

1980 Mathematics Subject Classification: 60K25, 68M20.

Key Words \& Phrases: M/G/1 queue, permanent customers, branching process, queue length, sojourn time.

\section{INTRODUCTION}

This study is devoted to a simple variant of the classical M/G/1 queue. It is an M/G/1 FCFS queue with two types of customers, viz. (i) ordinary customers who arrive according to a Poisson process, and (ii) permanent customers, who immediately return to the end of the queue after having received a service.

The main aim of the paper is to present a basic study of the influence of the $K$ permanent customers on queue length and sojourn time of the 'Poisson customers'. In particular it is shown, for the case that the service time distributions of the Poisson customers and all $K$ permanent customers are negative exponential with identical means, that the queue length and sojourn time distributions of the Poisson customers are the $(K+1)$-fold convolution of those for the case without permanent customers. This remarkable convolution property appears to be restricted to the exponential case.

The main reason for studying the influence of the permanent customers on the Poisson customers is that the underlying $M / G / 1$ variant exposes - stripped from all non-essential features - a structure that appears in many representations of computer and communication networks. Determining and understanding the influence of one class of customers on another one, is of theoretical interest and is useful for perceiving the operation of more complex queueing systems. Another reason for studying the model is that it has several interesting relations with other queueing models. These relations will be discussed in Section 3. Here it suffices to mention one example. For the special case of $K=1$ permanent customer, the reader will easily see the connection with a vacation queue, a queueing model where the server interrupts the service to a customer stream at certain epochs to take a vacation. This connection will be elaborated upon in Section 3. For general $K$ the model is a vacation queue of a type not studied before.

\section{Model description}

The model under consideration is a single server single queue model with two classes of customers, viz. (i) a class of ordinary customers who arrive according to a Poisson process with rate $\lambda$, and (ii) a class of $K$ permanent customers ( $K$ a fixed positive integer) who immediately return to the end of the queue after having received a service. The server serves the customers of the queue in first-come-firstserved order.

Report BS-R8919

Centre for Mathematics and Computer Science

P.O. Box 4079, 1009 AB Amsterdam, The Netherlands 
The service times of the Poisson customers and of the permanent (also called recurrent) customers are assumed to be independent stochastic variables; those of the Poisson customers all have the same distribution $B($.$) and those of the K$ recurrent customers all have the same distribution $B_{R}($.$) . We also$ introduce the following notation and assumptions:

$$
\begin{aligned}
& \beta(\rho):=\int_{0-}^{\infty} e^{-\rho t} d B(t), \quad \beta_{R}(\rho):=\int_{0-}^{\infty} e^{-\rho t} d B_{R}(t), \quad \operatorname{Re} \rho \geqslant 0, \\
& \beta:=\int_{0}^{\infty} t d B(t)<\infty, \quad \beta_{R}:=\int_{0}^{\infty} t d B_{R}(t)<\infty, \\
& a:=\lambda \beta<1, \quad a_{R}:=\lambda \beta_{R} .
\end{aligned}
$$

The composition of the queue is described as follows (cf. Fig. 1).

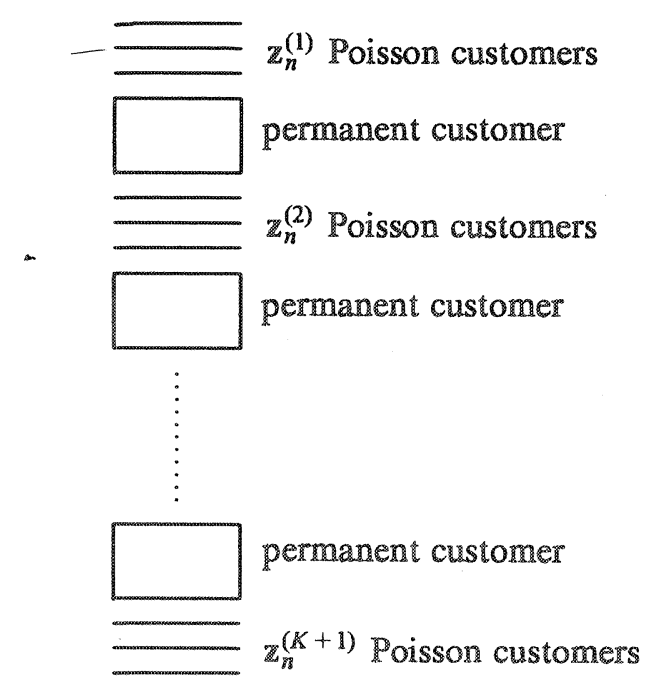

Figure 1

Counted from the head of the queue, at the nth service completion epoch there is a fixed group consisting of $\mathbb{z}_{n}^{(1)}$ Poisson customers, $\mathbb{z}_{n}^{(1)} \in\{0,1,2, \ldots\}, n=1,2, \ldots$; subsequently there is a recurrent customer, followed by a second group of $z_{n}^{(2)}$ Poisson customers, etc., until finally the queue is ended by a $(K+1)$ th group of $z_{n}^{(K+1)}$ Poisson customers. The assumption of independence of the service times of all customers, and the stochastic structure of the arrival process imply that $\left\{z_{n}^{(1)}, z_{n}^{(2)}, \ldots, \mathbb{z}_{n}^{(K+1)}, n=1,2, \ldots\right\}$ is a vector Markov chain. It can be shown that, for $a<1$, the vector Markov chain possesses a unique stationary distribution. We return to this issue in Section 2.

In that section we investigate the above described extension of the classical $M / G / 1$ queue, mainly concentrating on the vector Markov chain of queue lengths at service completion epochs and exploiting the close relationship with branching processes. The results appear to be interesting generalizations of queue length results for the ordinary $M / G / 1$ queue. The sojourn time distribution for Poisson customers will also be derived. Section 3 contains a few generalizations. Also, relations with vacation queues and cyclic server queues are pointed out. 


\section{ANALYSIS}

We start with the case of one recurrent customer, to reveal some of the basic features of the model and of its analysis without having to suffer from a large notational burden.

Let $\mathbf{X}_{n}$ denote the number of Poisson customers at the $n$th service completion epoch of the single recurrent customer, and let $b_{n}($.$) denote its generating function, n=1,2, \ldots$. Introduce for $|q| \leqslant 1, h=1,2, \ldots$

$$
\begin{aligned}
& \delta_{0}(q):=q \\
& \delta_{h+1}(q):=\beta\left\{\lambda\left(1-\delta_{h}(q)\right)\right\} .
\end{aligned}
$$

\section{THEOREM 2.1}

The Markov chain $\left\{\mathbf{X}_{n}, n=1,2, \ldots\right\}$ is positive recurrent iff $a<1$. For $a<1$, the generating function $b($. of the stationary distribution of the number of Poisson customers at a service completion of the single recurrent customer is given by

$$
b(q)=\prod_{h=0}^{\infty} \beta_{R}\left\{\lambda\left(1-\delta_{h}(q)\right)\right\},|q| \leqslant 1
$$

Proof

Relating the number of Poisson customers at the end of the $n$th and $(n+1)$-th service of the single recurrent customer, the following functional equation is easily seen to hold:

$$
b_{n+1}(q)=\beta_{R}\{\lambda(1-q)\} b_{n}(\beta\{\lambda(1-q)\}), \quad|q| \leqslant 1, \quad n=1,2, \ldots
$$

Differentiating both sides of this relation w.r.t. $q$ and letting $q \rightarrow 1$ it is seen that

$$
E \mathbf{X}_{n+1}=\lambda \beta E \mathbf{X}_{n}+\lambda \beta_{R}
$$

It follows that the Markov chain $\left\{\mathbf{X}_{n}, n=1,2, \ldots\right\}$ cannot be positive recurrent if $a=\lambda \beta \geqslant 1$. This was already obvious from the fact that, in the $M / G / 1$ queue without permanent customers, $a<1$ is a necessary condition for the existence of a stationary queue length distribution.

Using (2.1), one can iterate (2.3) in the following way:

$$
\begin{aligned}
b_{n+1}(q) & =\beta_{R}\left\{\lambda\left(1-\delta_{0}(q)\right)\right\} b_{n}\left(\delta_{1}(q)\right)=\beta_{R}\left\{\lambda\left(1-\delta_{0}(q)\right)\right\} \beta_{R}\left\{\lambda\left(1-\delta_{1}(q)\right)\right\} b_{n-1}\left(\delta_{2}(q)\right) \\
& =\cdots \\
& =\prod_{h=0}^{n-1} \beta_{R}\left\{\lambda\left(1-\delta_{h}(q)\right)\right\} b_{1}\left(\delta_{n}(q)\right) .
\end{aligned}
$$

It is well known from branching theory (cf. Harris [9], Theorem 4.1 of Chapter 1) that $\delta_{n}(q)$ is the generating function of a stochastic variable $\omega_{n} \in\{0,1,2, \ldots\}$,

$$
\delta_{n}(q)=E\left\{q^{\omega_{n}}\right\},|q| \leqslant 1,
$$


where $\omega_{n}$ is the offspring in the $n$th generation of a single ancestor in the 0th generation, with $\beta\{\lambda(1-q)\}$ the generating function of the branching distribution of a single element. In queueing terminology, $\omega_{1}$ is the number of Poisson arrivals during one service of a Poisson customer, and $\omega_{2}$ is the number of Poisson arrivals during the service of those $\omega_{1}$ customers, etc.

Because $E \omega_{1}=a<1$, this branching process extincts with probability one (cf. Harris [9], Theorem 6.1 of Chapter 1 ), and for $|q| \leqslant 1$,

$$
\lim _{n \rightarrow \infty} \delta_{n}(q)=1
$$

with $\delta_{n}(1)=1, n=0,1, \ldots$.

We are almost ready to let $n \rightarrow \infty$ in (2.4). We shall prove that the infinite product

$$
\text { IP }:=\prod_{h=0}^{\infty} \beta_{R}\left\{\lambda\left(1-\delta_{h}(q)\right)\right\}
$$

converges if $a<1$ (if the product of the first $n$ terms tends to zero for $\mathrm{n}$ tending to infinity, IP is said to diverge to zero). For simplicity, in the remainder of this proof $q$ is taken to be real, $-1 \leqslant q \leqslant 1$. The theory of infinite products, cf. Titchmarsh [11, Ch. 1], reveals that IP converges iff

$$
\sum_{h=0}^{\infty}\left[1-\beta_{R}\left\{\lambda\left(1-\delta_{h}(q)\right)\right\}\right]
$$

converges. For $a<1$,

$$
1-\beta_{R}\left\{\lambda\left(1-\delta_{h}(q)\right)\right\} \leqslant\left(1-\delta_{h}(q)\right) \lambda \beta_{R}
$$

Now

$$
1-\delta_{h}(q)=1-\beta\left\{\lambda\left(1-\delta_{h-1}(q)\right)\right\} \leqslant\left(1-\delta_{h-1}(q)\right) \lambda \beta \leqslant \cdots \leqslant(1-q)(\lambda \beta)^{h} .
$$

Combination of the last two formulas shows that IP converges. Hence, for $a<1$, the generating functions $b_{n}(q)$ converge for $n \rightarrow \infty$ to the function $b(q)$ given in $(2.2)$; and since $b(1)=1$ the continuity theorem for generating functions (cf. Feller [7]) implies that $b(q)$ is the generating function of the stationary distribution of the $\mathbb{X}_{n}$-process.

Note that, for $\beta_{R}(.) \equiv \beta(),. b(q)$ equals $\prod_{n=1}^{\infty} \delta_{n}(q)$.

We now turn to the case of $\mathbb{K}$ permanent customers, $K$ being an arbitrary positive integer. The same branching stochastic variables and generating function $b($.$) , and a similar recursion between$ generating functions as in (2.3), will lead to the determination of the multivariate generating function of the joint distribution of the queue lengths $\mathbb{z}_{n}^{(1)}, \ldots, \mathbb{z}_{n}^{(\mathbb{K}+1)}$. Some thought will convince the reader that, for any finite $\beta_{\mathbb{R}}$, the vector Markov chain $\left\{\mathbb{z}_{n}^{(1)}, \mathbb{Z}_{n}^{(2)}, \ldots, \mathbb{z}_{n}^{(K+1)}, n=1,2, \ldots\right\}$ possesses a unique stationary distribution iff $a<1$, regardless of the presence of the permanent customers. A proof could be given along the lines of the proof of Theorem 2.1 .

In the sequel it is assumed that $a<1$. Let $\left\{z^{(1)}, \ldots, z^{(K+1)}\right\}$ denote a vector with distribution the stationary distribution of the vector Markov chain $\left\{\mathrm{z}_{n}^{(1)}, \mathrm{z}_{n}^{(2)}, \ldots, \mathrm{z}_{n}^{(K+1)}, n=1,2, \ldots\right\}$. 
THEOREM 2.2

For $\left|q_{j}\right| \leqslant 1, j=1, \ldots, K+1$ :

$$
\begin{aligned}
& E\left[q_{1}^{z^{(1)}} \cdots q_{K+1}^{z^{(x+1)}}\right] \\
& \quad=\frac{1-a}{1-a+a_{R}} \frac{\beta\left\{\lambda\left(1-q_{K+1}\right)\right\} b\left(q_{K+1}\right) / \beta_{R}\left\{\lambda\left(1-q_{K+1}\right)\right\}-q_{1} b\left(q_{1}\right)}{\beta\left\{\lambda\left(1-q_{K+1}\right)\right\}-q_{1}} \prod_{j=2}^{K} b\left(q_{j}\right) .
\end{aligned}
$$

Proof

See the appendix.

\section{REMARK 2.1}

Apparently $\mathrm{z}^{(2)}, \ldots, \mathrm{z}^{(K)}$ are independent, identically distributed stochastic variables, which are moreover independent of $\mathrm{z}^{(1)}$ and $\mathrm{z}^{(K+1)}$; their generating function is $b($.$) . In retrospect, this should not be$ considered surprising. At a certain point in time a block of Poisson customers is 'completed', when a permanent customer joins the end of the queue; the length of this block subsequently becomes $z^{(K)}$, later on $\mathrm{z}^{(K-1)}, \mathrm{z}^{(K-2)}, \ldots, \mathrm{z}^{(2)}$, and the lengths of completed blocks are not influenced by blocks following it or yet to be completed. In Theorem 2.1 we have already shown, for $K=1$, that the generating function of the block length distribution is given by $b($.$) .$

$\mathrm{z}^{(1)}$ and $\mathrm{z}^{(K+1)}$ are correlated; their sum behaves similarly as the queue length in an ordinary $\mathrm{M} / \mathrm{G} / 1$ queue, apart from the periods in which a recurrent customer is in service.

Until now we have studied the joint distribution of the lengths of the blocks of Poisson customers at an arbitrary service completion epoch. We next concentrate on the departure epochs of Poisson customers, calculating the distribution of the total queue length $\mathbf{Z}$ at such departure epochs. An upand-down-crossing argument shows that this distribution also equals the total queue length distribution seen by an arriving Poisson customer; the PASTA property in turn implies that this is also the steady-state total queue length distribution of the Poisson customers.

LEMMA 2.1

$$
E\left[q^{\mathbb{Z}}\right]=\left[(1-a) \frac{(1-q) \beta\{\lambda(1-q)\}}{\beta\{\lambda(1-q)\}-q}\right] \frac{b^{K}(q)}{\beta_{R}\{\lambda(1-q)\}} \frac{1-\beta_{R}\{\lambda(1-q)\}}{a_{R}(1-q)} .
$$

\section{PROOF}

Denoting by $P$ the event that a departing customer is a Poisson customer, and by $\bar{P}$ its complement, it follows:

$$
E\left[q^{z^{(1)}+\ldots+z^{(x+1)}}\right]=E\left[q^{z^{(i)}+\ldots+z^{(x+1)}} \mid P\right] \operatorname{Pr}\{P\}+E\left[q^{z^{(i)}+\cdots+z^{(x+1)}} \mid \bar{P}\right] \operatorname{Pr}\{\bar{P}\}
$$

Using the facts that (see also (A.6)) $\operatorname{Pr}\{\bar{P}\}=(1-a) /\left(1-a+a_{R}\right)$ and that all 'completed' blocks of customers are independent, identically distributed stochastic variables with generating function $b($.$) , it$ follows that, for $|q| \leqslant 1$,

$E\left[q^{\mathbb{Z}}\right]=\frac{1-a+a_{R}}{a_{R}} E\left[q^{2^{(1)}+\ldots+z^{(R+1)}}\right]-\frac{1-a}{a_{R}} b^{K}(q)$

From (2.7) and (2.9), for $|q| \leqslant 1$, 


$$
E\left[q^{\mathbb{Z}}\right]=\frac{1-a}{a_{R}} b^{K}(q) \frac{\frac{\beta\{\lambda(1-q)\}}{\beta_{R}\{\lambda(1-q)\}}-\beta\{\lambda(1-q)\}}{\beta\{\lambda(1-q)\}-q},
$$

which can be rewritten as (2.8).

It should be noted that the term between square brackets in (2.8) represents the generating function of the steady-state queue length distribution in the $M / G / 1$ queue without permanent customers (note that it is not allowed to put $K=0$ in (2.8); in the derivation $K$ is explicitly assumed to be positive).

The following special results and corollaries are worth mentioning.

A. All service time distributions equal

If $B(.) \equiv B_{R}($.$) , then for |q| \leqslant 1$,

$$
E\left[q^{\mathbb{Z}}\right]=\left[(1-a) \frac{(1-q) \beta\{\lambda(1-q)\}}{\beta\{\lambda(1-q)\}-q}\right] \frac{b^{K}(q)}{\beta\{\lambda(1-q)\}} \frac{1-\beta\{\lambda(1-q)\}}{\beta \lambda(1-q)}
$$

B. All service time distributions equal and negative exponential

If $B(t)=B_{R}(t)=1-e^{-t / \beta}$, then, cf. (2.1),

$$
\delta_{h}(q)=\frac{1-a+a\left(1-a^{h-1}\right)(1-q)}{1-a+a\left(1-a^{h}\right)(1-q)}, \quad h=1,2, \ldots,
$$

and, cf. (2.2),

$$
b(q)=\frac{1-a}{1-a q}
$$

which equals the generating function of the queue length distribution in an ordinary $M / M / 1$ queue. From (2.10), for $|q| \leqslant 1$,

$$
E\left[q^{\mathbb{Z}}\right]=\left(\frac{1-a}{1-a q}\right)^{K+1}
$$

This result exposes the remarkable effect of the presence of permanent customers on the queue length of the Poisson customers in an M/M/1 queue: their queue length distribution becomes the $(K+1)$ fold convolution of the queue length distribution in the system without those permanent customers. This phenomenon was already observed by J.L. van den Berg in his investigations of queue lengths and sojourn times in feedback queues without and with permanent customers (see [2]); in fact his observation triggered the research reported on in the present paper. Formula (2.10) shows that the above-mentioned convolution phenomenon is an isolated one which only applies in the exponential case; in the case of general service time distributions one only observes a vacation-type decomposition of the queue length generating function.

C. Cycle times and sojourn times

Cycle times of permanent customers and sojourn times of Poisson customers are readily derived from 
the above queue length results. Indeed, one can use the classical observation for FCFS queues that the number of Poisson customers left behind by a departing customer equals the number of Poisson arrivals during the sojourn time of that departing customer.

The number of Poisson customers left behind after the departure of a permanent customer has generating function $b^{K}(q)$ (K complete blocks of Poisson customers are left behind), and hence, with $\mathrm{C}$ the time between two successive departures of a particular permanent customer,

$$
E[\exp \{-\lambda(1-q) C)\}]=b^{K}(q),|q| \leqslant 1,
$$

and

$$
E[\mathbf{C}]=\frac{K \beta_{R}}{1-a} .
$$

Similarly, with $\mathbf{S}$ the sojourn time of an arbitrary Poisson customer,

$$
E[\exp \{-\lambda(1-q) \mathbf{S}\}]=E\left[q^{\mathbb{Z}}\right],|q| \leqslant 1,
$$

with $E\left[q^{\mathbb{Z}}\right]$ given by (2.8); and with $\beta^{(2)}, \beta_{R}^{(2)}$ the second moment of the service time of a Poisson customer respectively a permanent customer:

$$
E[S]=\frac{\lambda \beta^{(2)}}{2(1-a)}+\beta+\frac{K \beta_{R}}{1-a}-\beta_{R}+\frac{\beta_{R}^{(2)}}{2 \beta_{R}}
$$

In the special case of identical, negative exponential, service time distributions, we obtain for $\operatorname{Re} \zeta \geqslant 0$ :

$$
\begin{aligned}
& E[\exp \{-\zeta \mathbf{C}\}]=\left(\frac{1-a}{1-a+\beta \zeta}\right)^{K} \\
& E[\exp \{-\zeta S\}]=\left(\frac{1-a}{1-a+\beta \zeta}\right)^{K+1}
\end{aligned}
$$

Note that in this exponential (product-form) case a departing (and hence again arriving) permanent customer sees the system in equilibrium with one less customer of his own type, which confirms the relation between (2.17) and (2.18). Also note that the presence of $K$ permanent customers leads to a $(K+1)$-fold increase of the sojourn times of the Poisson customers.

\section{GeNerALIZATIONS AND RELATED MODELS}

In this section we point at a few possible generalizations of the results in the previous section, and we show that our model bears a relation to several queueing models that have recently attracted attention.

\section{A. Generalizations}

The results of Section 2 can be generalized in several ways. Firstly, one could take different service time distributions for each of the permanent customers. Secondly, one may allow different arrival rates during the service of permanent customers and of Poisson customers. An interesting third possibility is that Poisson customers, after having obtained a service, are fed back to the end of the queue with probability $P$ (and leave the system with probability $1-P$ ). The details for some of these cases 
will be presented in [2]. In the feedback case, Formula (A.3) of the appendix remains valid when $\beta\{\lambda(1-q)\}$ is replaced by $(1-P+P q) \beta\{\lambda(1-q)\}$; similarly, the defining relations (2.1) for $\delta_{h}($. become

$$
\begin{aligned}
& \delta_{0}(q):=q \\
& \delta_{h+1}(q):=\left(1-P+P \delta_{h}(q)\right) \beta\left\{\lambda\left(1-\delta_{h}(q)\right)\right\} .
\end{aligned}
$$

Formula (2.7) is accordingly changed. The rationale behind the inclusion of feedback is that it leads to results for processor sharing queues. In a series of studies Van den Berg et al. (see in particular [2,3]) have presented an exact analysis of queue lengths and sojourn times in $M / M / 1$ queues with a general feedback mechanism. By simultaneously letting the mean service time at each loop go to zero and the feedback probabilities approach one in an appropriate way, those queue length and sojourn time results approach their counterpart for the $M / G / 1$ queue with processor sharing. This still holds when permanent customers are included. A study of queue lengths and sojourn times for an $M / G / 1$ queue with processor sharing and permanent customers is interesting both from a theoretical and a practical point of view. One example where such a queueing model may arise is provided by a Stored Program Control (SPC) telephone exchange which handles both call requests and operator tasks (De Waal [12]).

\section{B. Relations with other queueing models}

(i) Vacation queues

When $K=1$, the service times of the single permanent customer can be viewed as server vacations. The M/G/1 queue with one permanent customer behaves exactly like an M/G/1 queue with vacations and gated service. This is an $M / G / 1$ queue in which, after a server vacation, the server handles exactly those customers who are present at the end of the vacation, etc. The generating function of the queue length distribution in the $M / G / 1$ queue with vacations and gated service is given by (2.8) with $K=1$. Note that this formula exhibits the typical decomposition character of vacation queues, including the occurrence of the Laplace-Stieltjes transform of a residual vacation time. It is a special case of the three-way decomposition proven by Fuhrmann and Cooper [8] for vacation queues. For the case of the $M / G / 1$ queue with vacations and exhaustive service (the server continues serving until the queue is empty) the following decomposition result is well known. With $\mathbf{Z}_{v a c}$ the steady-state queue length in the $M / G / 1$ queue with vacations with Laplace-Stieltjes transform $\beta_{R}\{$.$\} , and with exhaus-$ tive service,

$$
E\left[q^{\mathrm{Z}_{\mathrm{wox}}}\right]=\left[(1-a) \frac{(1-q) \beta\{\lambda(1-q)\}}{\beta\{\lambda(1-q)\}-q}\right] \frac{1-\beta_{R}\{\lambda(1-q)\}}{a_{R}(1-q)} .
$$

This formula should be compared with (2.8).

(ii) Cyclic service models

The $M / G / 1$ queue with vacations is a special case of a polling model, a single-server multi-queue model in which the server attends to the queues in cyclic order. The M/G/1 queue with $K$ permanent customers can also be viewed as a polling model with $K$ queues - albeit a rather special one. The service times of the permanent customers correspond to the switchover times of the server between successive queues. To take into account that in the $M / G / 1$ model there really is only one queue, we have to assume that arrivals at a particular queue of the polling model are only possible during the server visit to that queue and during the subsequent switchover time; thereafter arrivals can only take place at the next queue, etc.

The resulting polling model is non-standard, but the extensive knowledge about polling models may still yield useful insight into the behaviour of the $M / G / 1$ queue with additional permanent customers. 
We mention one of the examples to be presented in [2]. Under very general conditions, the mean cycle time in a polling model is given by the sum of the mean switchover times, divided by one minus the load of the system. Hence the mean cycle time of a permanent customer in the $M / G / 1$ queue with $K$ additional customers with mean service times $\beta_{R}^{(1)}, \ldots, \beta_{R}^{(K)}$ is given by $\sum_{i=1}^{K} \beta_{R}^{(i)} /(1-a)$.

(iii) A service system with two stages of waiting

Ali and Neuts [1] consider an M/G/1 queue with a waiting room and a service room. Arriving customers first join the waiting room. Whenever the second stage of the queue, the service room, becomes empty it is replenished by the transfer of all customers from the waiting room and the addition of a positive random number of overhead customers (cf. the completion of a 'block' of customers in our model). In [1] these overhead customers are indistinguishable from the other customers. Customers whose service is completed may return to the waiting room with probability $P$. Ali and Neuts determine the stationary distributions of queue lengths and waiting times at various imbedded random epochs. The overhead customers give rise to a similar Galton-Watson branching process as in our study.

(iv) The finite and infinite source interaction model

In [10] Kaufman studies the following model of finite and infinite source interaction. Infinite source customers arrive at a single server queue according to a Poisson process, requiring negative exponentially distributed service times. There are $K$ finite sources, having negative exponentially distributed think times. The finite source customers require a negative exponentially distributed service time at the single server, and then return to the think phase, etc. The single server serves all customers in FCFS order. Kaufman presents a very accurate approximation method for estimating all mean performance measures of interest, thus obtaining insight into the extent to which the finite source customers increase the congestion caused by the Poisson customers. Kaufman's model reduces to the present one (with exponential service times) when think times are zero. An exact analysis of Kaufman's model for the special case of $K=1$ finite source has been presented by Doshi and Wong [6] and Boxma [4]; in [5] it is outlined how this model with one finite source can be completely analyzed in the case of general service time distributions. The approach involves a similar iteration procedure as the one in the present paper.

\section{ACKNOWLEDGMENT}

The authors are indebted to J.L. van den Berg for some interesting discussions.

\section{REFRRENCES}

1. ALI, O.M.E., NeUTS, M.F. (1984). A service system with two stages of waiting and feedback of customers, J. Appl. Probab. 21, 404-413.

2. VAN DEN BERG, J.L. (1990). Ph.D. Thesis, to appear.

3. VAN DEN BerG, J.L., BOXMA, O.J., GroENENDIJK, W.P. (1989). Sojourn times in the M/G/I queuse with deterministic feedback, Stochastic Models 5, 115-129.

4. BoXMA, O.J. (1986). A queueing model of finite and infinite source interaction, Oper. Res. Letters $5,245-254$.

5. BoxMA, O.J. (1986). Models of two queues - a few new views. In: O.J. Boxma, J.W. Cohen, H.C. Tijms (eds.). Teletraffic Analysis and Computer Performance Evaluation (North-Holland, Amsterdam) pp. 75-98.

6. DoshI, B.T., WoNG, W.S. (1987). Exact solution of a simple finite infinite source interaction model, Queueing Systems 2, 67-82.

7. FeLLER, W. (1968). An Introduction to Probability Theory and its Applications, Vol. I (Wiley, New York).

8. FuHRMANn, S.W., COOPER, R.B. (1985). Stochastic decompositions in the M/G/1 queue with generalized vacations, Oper. Res. 33, 1117-1129. 
9. HARRIS, Th.E. (1963). The Theory of Branching Processes (Springer, Berlin).

10. Kaufman, J.S. (1985). Finite and infinite source interactions. In: E. Gelenbe (ed.). Performance '84 (North-Holland, Amsterdam) pp. 345-359.

11. Trtchmarsh, E.C. (1968). The Theory of Functions. (Oxford University Press, Oxford; 2nd ed.).

12. De WAAL, P.R. (1989). Private communication. 
APPENDIX: PROOF OF THEOREM 2.2

Remembering the definition of the queue lengths $\mathrm{z}_{n}^{(j)}, j=1, \ldots, K+1$, of the $K+1$ blocks of Poisson customers just after a service completion (cf. Fig. 1), we can write:

$$
\begin{aligned}
& \text { if } \mathbf{z}_{n}^{(1)}>0 \text { then } \\
& \mathbf{z}_{n+1}^{(1)}=\mathbf{z}_{n}^{(1)}-1, \mathbf{z}_{n+1}^{(j)}=\mathrm{z}_{n}^{(j)}, j=2, \ldots, K, \mathbf{z}_{n+1}^{(K+1)}=\mathbf{z}_{n}^{(K+1)}+v_{n+1} ; \\
& \text { if } \mathbf{z}_{n}^{(1)}=0 \text { then } \\
& \mathbf{z}_{n+1}^{(j)}=\mathbf{z}_{n}^{(j+1)}, j=1, \ldots, K-1, \mathbf{z}_{n+1}^{(K)}=\mathbf{z}_{n}^{(K+1)}+\mu_{n+1}, \mathbf{z}_{n+1}^{(K+1)}=0,
\end{aligned}
$$

with $v_{n+1}$ the number of arriving Poisson customers during the $(n+1)$ th service if this service concerns a Poisson customer, whereas $\mu_{n+1}$ is the number of arriving Poisson customers during the $(n+1)$ th service if this service concerns a permanent customer. Obviously, with $|q| \leqslant 1$,

$$
\begin{aligned}
& E\left[q^{{⿱_{n}+1}_{1}}\right]=\beta\{\lambda(1-q)\}, \\
& E\left[q^{{\beta_{n+1}}_{1}}\right]=\beta_{R}\{\lambda(1-\bar{q})\}
\end{aligned}
$$

From (A.1) it follows for $n=1,2, \ldots,\left|q_{j}\right| \leqslant 1, j=1, \ldots, K+1$,

$$
\begin{aligned}
& E\left[q_{1}^{z_{s+1}^{(1)}} \cdots q_{K+1}^{z_{K+1}^{(k+1)}} \mid z^{(1)}, \ldots, z_{1}^{(K+1)}\right]
\end{aligned}
$$

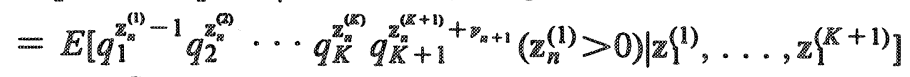

$$
\begin{aligned}
& +E\left[q_{1}^{z_{1}^{(2)}} \cdots q_{K}^{z_{n}^{(n)}} q_{K}^{z_{K}^{(k+1)}+\mu_{n+1}}\left(\mathbb{z}_{n}^{(1)}=0\right) \mid \mathbb{z}^{(1)}, \ldots, \mathbb{z}^{(K+1)}\right] .
\end{aligned}
$$

Denote by $\left\{\mathrm{z}^{(1)}, \ldots, \mathrm{z}^{(K+1)}\right\}$ a stochastic vector with distribution the stationary distribution of the process, it then follows from (A.2) for $\left|q_{j}\right| \leqslant 1, j=1, \ldots, K+1$ :

$$
\begin{aligned}
& E\left[q_{1}^{z^{(1)}} \cdots q_{K+1}^{z^{(x+1)}}\right]\left[1-\frac{\beta\left\{\lambda\left(1-q_{K+1}\right)\right\}}{q_{1}}\right] \\
& =-\frac{\beta\left\{\lambda\left(1-q_{K+1}\right)\right\}}{q_{1}} E\left[\left(\mathbb{z}^{(1)}=0\right) q_{2}^{z^{(2)}} \cdots q_{K+1}^{z^{(K+1)}}\right]+\beta_{R}\left\{\lambda\left(1-q_{K}\right)\right\} E\left[\left(\mathbb{z}^{(1)}=0\right) q_{1}^{z^{(x)}} \cdots q_{K}^{z^{(K+1)}}\right] .
\end{aligned}
$$

Taking $q_{1}=\beta\left\{\lambda\left(1-q_{K+1}\right)\right\}=\delta_{1}\left(q_{K+1}\right)$ to make the lefthand side of (A.3) zero, a standard analyticity argument yields:

$$
\begin{aligned}
& E\left[\left(\mathbb{Z}^{(1)}=0\right) q_{2}^{z^{(2)}} \cdots q_{K}^{q^{(x+1)}}\right] \\
& =\beta_{R}\left\{\lambda\left(1-q_{K}\right)\right\} E\left[\left(\mathbb{z}^{(1)}=0\right) \delta_{1}^{z^{(2)}}\left(q_{K+1}\right) q_{2}^{z^{())}} \cdots q_{K}^{z^{(X+1)}}\right] \\
& =\beta_{R}\left\{\lambda\left(1-q_{K}\right)\right\} \beta_{R}\left\{\lambda\left(1-q_{K-1}\right)\right\} E\left[\left(\mathbb{Z}^{(1)}=0\right) \delta_{1}^{z^{(2)}}\left(q_{K}\right) \delta_{1}^{z^{(9)}}\left(q_{K+1}\right) q_{2}^{z^{(9)}} \cdots q_{K-1}^{z^{(K+1)}}\right] \\
& =\beta_{R}\left\{\lambda\left(1-\delta_{1}\left(q_{K}+1\right)\right)\right\} \prod_{j=2}^{K} \beta_{R}\left\{\lambda\left(1-q_{j}\right)\right\} E\left[\left(z^{(1)}=0\right) \delta_{1}^{z^{(2)}}\left(q_{2}\right) \delta_{1}^{z^{(3)}}\left(q_{3}\right) \cdots \delta_{1}^{z^{(K+1)}}\left(q_{K+1}\right)\right] \text {. }
\end{aligned}
$$

A similar iteration procedure as was used in (2.4) leads to: 
12

$$
\begin{aligned}
& E\left[\left(\mathbb{z}^{(1)}=0\right) q_{2}^{z^{(x)}} \cdots q_{K+1}^{z^{(x+1)}}\right]=\prod_{h=0}^{m} \beta_{R}\left\{\lambda\left(1-\delta_{h+1}\left(q_{K+1}\right)\right)\right\} \\
& \prod_{j=2}^{K} \prod_{h=0}^{m} \beta_{R}\left\{\lambda\left(1-\delta_{h}\left(q_{j}\right)\right)\right\} E\left[\left(\mathbb{z}^{(1)}=0\right) \delta_{m+1}^{z^{()}}\left(q_{2}\right) \cdots \delta_{m+1}^{z^{(x+1)}}\left(q_{K+1}\right)\right]
\end{aligned}
$$

Using (2.2) and (2.6) it follows that

$$
\begin{aligned}
& E\left[\left(\mathrm{z}^{(1)}=0\right) q_{2}^{z^{(2)}} \cdots q_{K}^{z^{(++1)}}\right] \\
& =\operatorname{Pr}\left\{\mathrm{z}^{(1)}=0\right\}\left\{\prod_{j=2}^{K+1} b\left(q_{j}\right)\right\} / \beta_{R}\left\{\lambda\left(1-q_{K+1}\right)\right\} .
\end{aligned}
$$

Taking $q_{1}=\cdots=q_{K+1}=q$ in (A.3) and letting $q \rightarrow 1$, it follows that

$$
\operatorname{Pr}\left\{\mathrm{z}^{(1)}=0\right\}=\frac{1-a}{1-a+a_{R}} .
$$

Combination of (A.3), (A.5) and (A.6) yields the statement of the theorem. 